\title{
Central Venous Catheter-Related Bloodstream Infection and Cryptococcus neoformans
}

\author{
Felipe F. Tuon², Hugo M. P. Morales ${ }^{1}$, Sergio R. Penteado-Filho ${ }^{1,2}$, Margarete M. da-Silva ${ }^{3}$, Isabel de Quadros ${ }^{4}$ and Amina El \\ Hamoui ${ }^{4}$ \\ ${ }^{1}$ Division of Internal Medicine,Hospital Universitario Evangelico de Curitiba; ${ }^{2}$ Division of Infectious and Parasitic Diseases, Hospital \\ Universitario Evangelico de Curitiba; ${ }^{3}$ Division of Nephrology, Hospital Universitário Evangélico de Curitiba; ${ }^{4}$ Laboratory of Microbiology, \\ Hospital Universitário Evangélico de Curitiba;Curitiba, PR, Brazil
}

\begin{abstract}
This is the first case reported of central venous catheter-related fungemia due to $C$. neoformans. A patient with chronic renal failure developed a fungemia during the treatment of a dialysis-associated bacteremia. Cryptococcus neoformans grew in the catheter tip and blood culture. We addressed questions about this catheter-related fungemia. Key-Words: Cryptococcus neoformans, fungemia, central venous catheter.
\end{abstract}

Cryptococcal disease is a fungal infection which affects mostly immunosuppressed patients and is caused by Cryptococcus neoformans. Pulmonary and central nervous system are the most common sites of cryptococcal infection [1], moreover fungemia and cutaneous lesions have been described [2].

Cryptococcus laurentii, a different species of this genus, has been described as a agent of central venous catheter-related fungemia, however, C. neoformans was never described in central lines [3]. The aim of this case was to describe a possible case of catheter-related fungemia caused by $C$. neoformans in a patient with chronic renal failure.

\section{Case Report}

A 60-year-old woman was admitted in July 3, 2008 with signs and symptoms of bacteremia during hemodialysis. She was under hemodialysis since 2004 due to chronic renal failure secondary to systemic arterial hypertension. Laboratory findings showed a normal leukocyte count (3,530 K/dL), hemoglobin and platelet count of $6.3 \mathrm{~g} / \mathrm{L}$ and $137 \mathrm{~K} / \mathrm{dL}$, respectively. Vancomycin and ceftazidime were initiated after two blood culture samples and catheter removal. Blood cultures were negative and clinical conditions improved 72 hours after antibiotic introduction. The patient was asymptomatic with plan of discharge, but in the 16th day of admission the patient initiated with fever during hemodialysis, and new blood cultures were collected with catheter removal after blood samples. Laboratory findings showed a leukocyte count of $3,310 \mathrm{~K} / \mathrm{dL}$, hemoglobin count of $8.0 \mathrm{~g} / \mathrm{L}$ and platelet count of $80 \mathrm{~K} / \mathrm{dL}$. The patient was well and no therapy was introduced. The blood and catheter tip culture revealed Cryptococcus neoformans, confirmed by API (Biomerieux ${ }^{\circledR}$, France 821885202).

Received on 30 May 2009; revised 21 July 2009.

Address for correspondence: Dr. Felipe Francisco Tuon. Infectious and Parasitic Diseases Clinic, Hospital Universitário Evangélico de Curitiba, Alameda Augusto Stellfeld 1908, 8 . andar - SCIH - Bigorrilho, CEP number 80730-150, Curitiba, Brazil. Email: flptuon@gmail.com. Telephone: 5541 32405055. Fax: 554132405274.

The Brazilian Journal of Infectious Diseases 2009;13(4):317-318. (C) 2009 by The Brazilian Journal of Infectious Diseases and Contexto Publishing. All rights reserved.
Considering the diagnosis of fungemia, amphotericin B $0.5 \mathrm{mg} / \mathrm{kg} /$ day was initiated three days after blood culture sample, with clinical improvement within 2 weeks of treatment. After 4 weeks, the patient died with septic shock due to Klebsiella pneumoniae. In vitro antifungal susceptibility tests were not performed as well as molecular test comparing the strains from catheter and blood culture.

\section{Discussion}

Several cases of cryptococcal fungemia have been described. Catheter-related must be a very rare finding. A systematic search was performed on three medical databases (MEDLINE, LILACS and EMBASE), but only cases of $C$. laurentii catheter-related fungemia have been cited. In some reviews of fungemia, Cryptococcus have been included in the statistics, but without species identification [4]. Cryptococcus colonization of central venous lines probably has been found in several hospitals without publication.

API 20C was used for species identification. This test can differentiate $C$. neoformans from $C$. albidus and $C$. laurentii [5]. Nevertheless, API 20C cannot identify varieties of C. neoformans: C. neoformans var. gattii and C. neoformans var. neoformans. Candida catheter-related fungemia is well described. The risk of Cryptococcus misdiagnosed with API 20C is probably impossible. Furthermore, two different samples (catheter and blood) showed the same result.

In the case reported, the catheter colonization could be from skin or during fungemia. C. neoformans is environmental yeast-like, but some cases have been reported in the nosocomial section. A recent outbreak of Candida parapsilosis occurred in our nephrology division supposedly associated with pigeons. However, this association was discharged after feces exam from several pigeons, and Cryptococcus was not found too (data not showed). Environmental samples were collected in the same period of this outbreak and the case reported above, but no yeast was found.

Fungemia with further contamination/colonization of the catheter is the most reasonable theory. This patient probably reactivated a primary disease (lung) due to underlying immunosuppression related to chronic renal failure. 
The intravenous catheter was a disposal which could be hosted this yeast during fungemia.

It raises another doubt, whether the catheter was really colonized or just contaminated with infected blood. Cryptococcus neoformans collectively forms biofilms on polystyrene plates and medical devices [6]. Martinez and Casadevall demonstrated that fungal biofilm formation is dependent on support surface characteristics and that growth in the biofilm state makes fungal cells less susceptible to potential environmental stresses, including antibiotics [7;8].

The time needed for a biofilm formation is unknown and the possibility of a planktonic isolation during fungemia must be considered.

The outcome of this patient was not attributed to fungemia but increased the hospital permanency. This fact increased the risk of bacterial infection and indirectly increases the mortality.

Considering these facts, central venous catheter-related Cryptococcus neoformans bloodstream infection is a possible condition that can be more frequent than reported. The biofilm formation is an explanation to catheter-related infection and fungemia. Our case arise the theory of catheter related fungemia but contamination of the catheter cannot be discharged. Experimental studies should be performed to confirm this possibility.

\section{References}

1. Leite A.G., Vidal J.E., Bonasser F.F., Nogueira R.S., Oliveira A.C. Cerebral infarction related to cryptococcal meningitis in an HIV-infected patient: case report and literature review. Braz J Infect Dis 2004; 8: 175-9.

2. Pasqualotto A.C., Bittencourt S.C., de Mattos O.F., Severo L.C. Cryptococcemia. An analysis of 28 cases with emphasis on the clinical outcome and its etiologic agent. Rev Iberoam Micol 2004; 21: 143-6.

3. Johnson L.B., Bradley S.F., Kauffman C.A. Fungaemia due to Cryptococcus laurentii and a review of non-neoformans cryptococcaemia. Mycoses 1998; 41: 277-80.

4. Sota M., Ezpeleta C., Cisterna R. Description of 165 episodies of fungemia: a multicenter study. Rev Iberoam Micol 1999; 16: $30-5$.

5. Smith M.B., Dunklee D., Vu H., Woods G.L. Comparative performance of the RapID Yeast Plus System and the API 20C AUX Clinical Yeast System. J Clin Microbiol 1999; 37: 26978.

6. Martinez L.R., Ibom D.C., Casadevall A., Fries B.C. Characterization of Phenotypic Switching in Cryptococcus neoformans Biofilms. Mycopathologia 2008; 166: 175-80.

7. Martinez L.R., Casadevall A. Cryptococcus neoformans biofilm formation depends on surface support and carbon source and reduces fungal cell susceptibility to heat, cold, and UV light. Appl Environ Microbiol 2007; 73: 4592-601.

8. Martinez L.R., Casadevall A. Cryptococcus neoformans cells in biofilms are less susceptible than planktonic cells to antimicrobial molecules produced by the innate immune system. Infect Immun 2006; 74: 6118-23. 